Cuad. invest. hist. Brocar n. 16 (1990). Págs. 195-206.

\title{
PROCESO HISTORICO DE COLL DE LADRONES. DE FELIPE II A ALFONSO XII Y A LA SEGUNDA GUERRA MUNDIAL
}

\author{
Juan F. Esteban Lorente
}

En estas II Jornadas de Arte Riojano hemos preferido presentar este tema, incluido dentro de las generalidades de la INICIACION A LA ARQUITECTURA MILITAR, por cerrar con el mundo actual este ciclo que se inicia en los siglos IX y X, y por ser frecuentemente olvidados estos espectaculares castillos de la Edad Contemporánea.

Iniciamos el proceso de estudio de las fortificaciones alli donde normalmente se terminan los estudios de las fortalezas, con las preparadas para artillería de avancarga, las del tipo del ingeniero militar francés Vaubant.

La documentación próxima a estas fortificaciones de los siglos XIX y XX se encuentra recogía en los archivos de las Comandancias de Obras respectivas, teniendo en cuenta que además se encuentra una copia de ellas en el Servicio Histórico Militar y Servicio Geográfico del Ejército en Madrid, y otra en el Archivo General Militar de Segovia. En nuestro caso ofrecemos un resumen cronológico de la documentación conservada en el archivo de la Comandancia de Obras de Zaragoza ${ }^{1}$.

\section{COLL DE LADRONES. SituACióN}

El fuerte de Coll de Ladrones es el centro de un conjunto defensivo formado por dos edificios de acuartelameitno y una serie de galerías subterráneas para cañones y fusilería. La obra actual fue construida en los últimos años del siglo XIX. Está situado en lo alto de un cerro rocoso que limita verticalmente la confluencia del barranco de Izas con el río Aragón, justo al Norte de la actual estación de Canfranc. Es el punto ideal para cerrar el paso de la carretera, entonces recién terminada, que accede desde la frontera

1 De la riqueza de este archivo dimos cuenta en una exposición que organizamos con motivo del IV COLOQUIO DE ARTE ARAGONES, Zaragoza, octubre, 1989, Proyectos de arquitectura militar en Aragón en torno a 1900, el catálogo de la misma se publico, por el que suscribe y Francisco Julio Robres Uriol, en ARTIGRAMIA, n. 5 (1988), 315-337. 
francesa y se escogió porque su situación se encuentra en el límite prudente de comunicación con la población de Canfranc, en casos de problemas de nevadas.

La posición con ser buena tiene por delante, al Norte, dos montículos dominantes, de los cuales uno es muy peligroso pues su acceso por el lado norte es facil y queda oculto a las vistas desde Coll de Ladrones, éste es el montículo de La Sagüeta, en el que, como apoyo a Coll de Ladrones, se proyectará una pequeña batería dirigida directamente al paso de la frontera. Para garantizar las comunicaciones entre Coll de Ladrones y el pueblo de Canfranc, carretera hacia Jaca, se proyectaron dos torres de fusilería intermedias entre el pueblo y la fortificación; de este modo el complejo de la fortificación y defensa de la frontera española por el tradicional paso de Somport se centra en Coll de Ladrones con una avanzada y una retaguardia protegidas. En estos momentos en los que la artillería ha adquirido gran poder destructivo y se le preveía aún mayor en el futuro, la defensa contra ella se confía a la roca y al subterráneo, por ello la gran obra de estas fortificaciones son los demontes, túneles, colocación de los edificios en lugares protegidos por la roca de las posibles trayectorias de los obuses, desenfiladas, recios muros que puedan sostener bóvedas de cañón de $1 \mathrm{~m}$. de espesor, construidas en cemento, y sobre ellas un blindaje de más de tres metros de tierra apisonada, etc.

\section{Justificación histórica}

Diversas consideraciones impulsaron al gobierno de Alfonso XII y al subsiguiente de la regencia de Maria Cristina a abordar esta costosa política de defensa de la frontera del Pirineo, y entre todas ellas la que, al parecer, más pesó fue la memoria de la anterior invasión francesa, la conciencia de otra intentona similar, la mayor potencia militar francesa, y las fortalezas que similarmente habia ya construido Francia en la otra vertiente de los Pirineos, como lo de Urdox, que tanto se veían como defensivas como posibles puntos de iniciación ofensiva hacia nuestro país.

Este es el pensamiento contemporáneo que vemos reflejado en los memoriales y estudios militares que en el momento se hacen como el del Primer Teniente de Infantería don Enrique NAVARRO ABUJA en su conferencia pronunciada en la noche del viernes 24 de febrero de 1905, en el Centro del Ejército y de la Armada, Estudio geográfico-militar de los Pirineos aragoneses, Madrid, R. Velasco Imp., 1905, y ya lo había destacado el Teniente Coronel de E. M. don Francisco LARREA LISO en su memoria de 1894, Importancia estratégica actual de Aragón y en particular de Zaragoza, Zaragoza, tip. de Julián Sanz y Navarro, 1895 (2. ed).

\section{COLL DE LADRONES. RESUMEN HISTÓRICO}

\section{Siglos XVI y XVII}

Anteriormente a Felipe II existió en Canfranc un castillo, en la salida norte del pueblo; cerrando el acceso desde Francia. Otra pequeña fortaleza hubo en Candanchu que se usaba para vigilar y cobrar el paso; alli estuvo situado el hospital de Santa 
Cristina cuyos monjes se trasladaron a Jaca en 1558 , cuando el castillo ya estaba arruinado.

$\mathrm{El}$ interés por fortificar esta frontera contra los hugonotes franceses del Bearn se reinicia en 1592. Tras las “alteraciones de Aragón”, con la presencia en Jaca del general don Alonso de Vargas y de su comendador (verdadero Estado Mayor) don Tiburcio Spanochi, quien es el encargado de los informes y proceder para las fortificaciones del Pirineo.

En 1592 Spanochi cita el castillo arruinado de Candanchú, para el que nada se prevee; en el castillo de Canfrac "de buena fébrica" se recomienda su acondicionamiento para 25 o 30 soldados, como así se hizo; advierte también la conveniencia de edificar una torre vigia en el lugar "del puente de Roldán, en el puesto de Coll de Ladrones y la Cuca donde se estrecha el paso" (Oset Moreno, Enrique: El castillo de San Pedro de Jaca, Jaca, C.A.Z.A.R., 1971, p. 199), esta torre también se hizo y ya estaba terminada en 1641 cuando se la llama "Torre de la Spelunca", que tenia un alferez y 8 soldados (iden p. 233).

Por esas fechas aún no se había terminado la ciudadela de Jaca, llamada Castillo de San Pedro, que se inició tras varios informes, entre ellos los de Spanochi y sus planos, en 1596; se debió terminar en la segunda mitad del siglo XVII, a finales del reinado de Felipe IV, ya que su escudo adorna la puerta.

En la primera mitad del siglo XVIII esta ciudadela recibe obras de mejora en las fortificaciones, acuartelamientos e iglesia. Actividades estas que corresponden al programa de fortificación militar que llevó a cabo en toda España el gobierno de Felipe V.

En este programa del segundo cuarto del siglo XVIII es cuando se ejecutó una fortaleza moderna en el alto de Coll de Ladrones y se acondicionó la antigua torre de la Espelunca para una batería de cañones.

\section{COLL DE LADRONES EN EL SIGLO XVIII}

Conocemos la estructura del fuerte construido en el siglo XVIII gracias a unos planos ejecutados en 1878,1879 y 1888 .

Construido sobre la superficie de la roca en lo alto del cerro cubria el perímetro del monte, tenía su frente de acceso al Este, único lugar accesible y se adelantaba en forma de tenaza con una "luneta" central, la estructura del frente era similar a las cabezas de puente que se habían usado antes en la Guerra de Sucesión, u otros fuertes similares que sólo tuvieron un punto vulnerable, tal la ciudalela de Santiago en Manila, el frente del fuerte de los Capuchinos en Gerona, el de San Andrés en Orán, o los laterales del de San Fernando en Figueras, cualquiera de los costados de las ciudadelas de Barcelona o Pamplona etc. (CAPEL, H., SANCHEZ, J. E. Y MONCADA, O.: De Palas a Minerva. La formación científica y la estructura institucional de los ingenieros militares enel siglo XVIII, Madrid, C.S.I.C./Serbal, 1988).

En el caso de Coll de Ladrones la entrada estaba muchos más protegida que en todos los demás conocidos de la época, pues el camino, que venía frontal a la luneta, tenía que 
recorrerla por un lado para entrar por el foso tras doble puerta y paso por detrás de la luneta y entonces entrar por el centro de la tenaza.

El interior era una serie de 6 galerías cubiertas con bóveda de cañón dirigidas de Este a Oeste; se distribuyeron tres a cada lado de una calle central. Las 4 galerías exteriores, de unos 25 x 5 metros para los servicios de tropa con entrada única por el lado oeste; las colindantes a la calle, simétricamente, se compartimentaron para las oficinas y mando de la fortificación, las entradas daban a la calle y a la plaza del Oeste. En la galería del lado norte se abrieron dos vanos, en arco rebajado, emplazados en alto sobre el suelo de la galería, quizá para dos pequeños cañones que apuntaban al camino que viene de Francia y al montículo de Sasot. La parte superior era aterrazada útil para la artillería de montaña de la época.

\section{El siglo XIX}

Por unos memoriales del último tercio del siglo XIX sabemos que en 1815 el ingeniero español Verdejo hizo un proyecto para fortificar Coll de Ladrones, que no se tuvo en cuenta, y que unos años más tarde en 1823 al retirarse los franceses desmontaron el fuerte de Coll de Ladrones, arruinando a su vez, sin duda, la batería de la Espelunca.

Terminada la carretera a Francia por este paso de Canfranc y Candanchú, se inician los proyectos para la construcción de un nuevo fuerte en Coll de Ladrones, de acuerdo a la moderna técnica de fortificaciones y de artillería, con previsiones de futuro ya inciertas en aquellos años, dada la constante evolución de las armas.

1864 hizo una memoria el capitán de la brigada Francisco Rizzo, que en 1888 será Brigadier Comandante General Subinspector a quien competía el exámen de los proyectos de la comandnacia de ingenieros de Jaca.

1876 el T.C. Comandante Capitán José San Gil hizo un ante-proyecto de defensa del trozo de carretera de primer orden comprendido entre Canfranc y la frontera, que tuvo que reformar en 1877.

1877, 1 de diciembre, el mismo José San Gil hace el proyecto para dos torres de fusilería, que se deberán construir junto a la carretera, para completar y apoyar al fuerte de Coll de Ladrones. Fue aprobado por R.O. del 19 de marzo de 1878.

1878 el mismo ingeniero hizo el proyecto de desmonte para la instalación del fuerte; el proyecto del camino de acceso desde Canfranc a Coll de Ladrones, proyecto que, aprobado por R.O. de 20 de marzo de 1878 , va a ser reformado en 1879 , y tres veces dentro del año de 1884 , mientras se construye.

1879,13 de enero el comandante capitán Julio Rodriguez hace el anteproyecto para la construcción del fuerte de Coll de Ladrones; este es el documento de partida de todos los siguientes proyectos o reformas para la construcción del fuerte, sus edificios etc. En este año se trabaja en la carretera y en la construcción de la Torre n. ${ }^{\circ} 2$. El 23 de septiembre del mismo año, el mencionado Julio Rodríguez reforma el proyecto; esta reforma se debe a adjuntar detalles de medidas que no se habían podido tomar en enero, por causa de la nieve y sobre todo a tener que ajustar el proyecto a unas prevenciones que por oficio del 23 de mayo había hecho el Director General, las cuales, esencialmente, consistían en retrasar el frente de ataque reduciendo el espacio edificable. 
1880, el 13 de enero se aprueba el anteproyecto, pero el 29 de diciembre de ese año el Director General del Cuerpo, Carlos Berdugo, a quien competía el examen de los proyectos, envía unas instrucciones para reducir las obras y la guarnición concebida ya que se ha aprobado la construcción de otro fuerte en la Sagüeta como complemento avanzado de Coll de Ladrones. En ese año de 1880, el 30 de abril el mismo ingeniero hace el proyecto de desmonte para la instalación del fuerte.

1881,5 de marzo el ingeniero Julio Rodriguez hace un nuevo anteproyecto de construcción del fuerte de Coll de Ladrones. El 28 de mayo de este año se aprueba el anteproyecto y el proyecto de fortificación de la Sagüeta.

1883 se construye la Torre n. ${ }^{\circ} 1$ con el proyecto aprobado en la R.O. del 28 de Junio de 1882 .

1888, 3 de abril, el comandante capitán Julio Rodríguez elabora el proyecto de construcción del fuerte; el que puede considerarse definitivo y al que se le aplicarán las reformas subsiguientes. Por R.O. del 4 de agosto de 1888 es aprobado el proyecto. En ese año se había realizado ya la carretera de comunicación, los desmontes y túneles del fuerte, la galería aspillerada labrada en la roca, los muros de la caponera y sus bóvedas, los muros del frente de ataque, así como parte de los muros del cuartel. Se pide aumento de presupuesto y se prevee la posibilidad de instalar telégrafo por hilos u óptico y terminar las obras en dos campañas de 6 meses.

1890,31 de mayo, el presupuesto será ampliado y aprobado en agosto de ese año.

1892,8 de agosto, se suspenden las obras en la fortificación de la Sagüeta.

1893, 14 de mayo, se reforma el presupuesto; fue aprobado por R.O. del 14 de enero de 1895. Las obras no se habían concluido.

1894, 31 de julio, reforma adicional para la instalación de un cañón de tiro rápido en pozo y cúpula.

1896, 31 de enero, proyecto de consolidación del desvio del camino; aprobado por R.O. del 8 de abril del mismo año.

\section{El siglo $X X$}

1900, en noviembre se terminan las obras y en memoria de ello se puso la inscripción de la puerta: FUERTE/DE/COLL DE LADRONES/MCM.

1900,31 de diciembre, proyecto para la instalación del horno de pan, aprobado por R.O. del 25 de febrero de 1901.

1901, 18 de octubre, proyecto para reparación de la Torre n.․ 1 , destruida por un incendio el día 19 de marzo de 1900. Aprobado por R.O. del 9 de enero de 1902.

1902, 21 de junio, proyecto de instalación del material fijo de arillería en las casamatas. Aprobado por R.O. del 1 de septiembre de 1902. En este proyecto es cuando se plantean los accesorios metálicos como verjas y puente corredizo. La artillería no se termina de instalar hasta 1906.

1903 fue visitado por El rey Alfonso XIII, memoria de ello se guarda en una lápida de la batería inferior de la galería que dice: 
JUAN F. ESTEBAN

\section{S.M. EL REY D. ALFONSO XIII \\ A.A. REALES PRINCIPES DE ASTURIAS \\ VISITARON ESTA BATERIA \\ EL 5 DE SEPTIEMBRE DE}

1903

1904, 10 de febrero, proyecto para instalación de una batería de 4 obuses y 4 morteros inmediata al fuerte, en el collado, $y$ un edificio para cantina. Aprobado por R.O. del 20 de abril del mismo año.

1905,30 de abril aprobado un proyecto para una casilla de camineros para el cuidado del camino.

1906, 21 de marzo, proyecto para el almacén de efectos de administración.

1909, 21 de noviembre, proyecto para una casilla de peón caminero, aprobado por R.O. del 28 de enero de 1910.

1910, 2 de junio, por R.O. se dispone la demolición de la Torre $\mathbf{n} .{ }^{\mathrm{o}} \mathbf{2}$ con motivo de la construcción de la estación de ferrocarril de Canfranc. Se dispone la construcción de la batería de Samán en su sustitución.

1911, 23 de octubre por R.O. se proponen nuevos proyectos para acondicionar el fuerte de La Sagüeta. No se llegó a armar ni a terminar.

1911, 22 de diciembre, por R.O. se aprueba un proyecto para modificación de los parapetos y terraplenes.

1918, 31 de octubre, proyecto de reparación de la Torre $\mathrm{n} .{ }^{\circ} 1$.

1920, 1 de diciembre, proyecto para la instalación de cocinas "Preckler" en Coll de Ladromes.

1921. De hacia esta fecha debe ser un proyecto para la construcción del observatorio en el fuerte ( $\sin$ fecha).

1927, 18 de marzo, se aprueba el presupuesto de reparación de cubiertas del fuerte.

1929, 5 de febrero, proyecto para nueva instlación de teléfono, telégrafo, luz eléctrica y reparación de la conducción de agua.

1934, 27 de junio, por orden del Ministerio de Guerra se entrega al Ministerio de Hacienda la Torre $n .{ }^{\circ}$, el día 13 de septiembre.

$1942,1944,1946,1948,1949,1951,1952,1953$ y 1955 reparaciones de mantenimiento en el fuerte de Coll de Ladrones.

1949, 15 de junio, proyectos para las obras de fortificación del Pirineo. Coll de Ladrones es la zona N. de R. 112.

1950, 31 de mayo otros proyectos del mismo tipo. Como consecuencia de ellos se instalarán en el monte: Refugios en caverna, ovservatorios, emplazamientos para fusil ametrallador, emplazamientos para ametralladora y emplazamientos para mortero de $81 \mathrm{~mm}$.

1960-61 se clausura el Fuerte de Coll de Ladrones y se ejecutan obras para dejarlo cerrado.

1964, como había sido usado para diversas ocupaciones, entre ellas el ganado, se hacen reparaciones extrictamente indispensables; se desaconseja su reparación pero se hace amplia información de su estado y de las obras necesarias para su conservación. 


\section{Coll de LADRones. Proceso de la construcción}

Para la construcción del nuevo fuerte hubo que demoler el fuerte anterior y vaciar el montículo rocoso; el suelo para edificar se dejó entre 16 y 20 metros por debajo de la plataforma superior del fuerte anterior. Se respetó como protección la parte norte de la roca y la galería de piedra que esta construida sobre ella, la cual se rellenó de tierras; asi se dejó un escudo de piedra matrual entre 10 y $17 \mathrm{~m}$. de espesor, protegidos por este escudo se construyeron dos edificios, cuyo nivel de techumbres se calcularon ligeramente escalonadas. El edificio más próximo al muro de piedra fue destinado para cuartel, tanto de la tropa de artillería (50) como de la infantería (150), en total 200 soldados, con sus armas y pertrechos personales; es un edificio longitudinal de dos naves y dos pisos, la entrada mira directamente a la entrada del fuerte. El otro edificio, con un frente más noble y situado en el lateral sur, fue dedicado a enfermería y a los "pabellones", alojamiento de los oficiales con sus subalternos a las oficinas y al mando, éste en el piso superior. Se hizo un tercer edificio, pequeño, en el extremo oeste destinado a letrinas. Todos estos edificios se forraron al exterior en sillarejo de piedra del país, muy bien labrada, y las esquinas, impostas, marcos de puertas y vantanas se ejecutan en sillería algo mayor; ornamentalmente se diferenció, muy discretamente, el edificio de pabellones de los demás; sólamente se destacó la sillería de las esquinas con pequeños encadenados, los marcos de las ventanas y puerta son más visibles y la pieza de prestigio fue una escalera exterior de doble tramo para acceder al piso de mando.

El murallón rocoso del norte se perforó con cuatro túneles destinados a sendas piezas de artilléria y un quinto como polvorín de la artillería.

El extremo sur del monte rocoso se perforó con una galería, en descenso, para fusiles, que protegen y atacan la carretera de acceso al fuerte; esta galería termina, al oeste, en una casamata done si instaló una batería de tiro rápido dirigida para bloquear la carretera nacional; al inicio de la galería un pequeño tunel se destinó a polvorín de la infanteria. Esta "galería aspillerada" fue en su momento una de las obras más difíciles y de mayor éxito en la construcción.

En la parte oeste de la superficie del montículo sólo se construyó un parapeto, para cerrar el espacio, ya que el monte cae verticalmente sobre el río.

El lado este es el de acceso natural y a él va a desembocar no sólo la carretera de acceso que hubo de construirse sino el camino que desde Sallent de Gállego accede a Canfranc, camino de Izas. Esta zona se le conoce con el nombre de "frente de ataque". Para mejorar este frente se desmontó una pequeña ondulación situada en el collado que obstaculizaba la vista del camino de Izas, se construyó un "glacis", picando la roca, cubierto por la artilleria y fusiles del muro del frente de ataque; en este glacis se abrió una zanja central que termina en tunel, es la puerta de entrada a la que le precede un pequeño foso con agua. Puerta y foso se franqueaban con un puente sobre railes, al otro lado se levanta el muro del frente de ataque. Haciendo codo y girando a la derecha se penetraba en el foso de entrada protegido por varias cañoneras y fusilería así como una "caponera" (casamata con cañones y fusiles dispuesta en codo para proteger las entradas); junto a la caponera está la puerta de acceso al fuerte, con su puerta blindada; el foso continúa para terminar en el vacio del lado norte. 
El muro del frente de ataque está perforado por varios túneles que albergan el cuerpo de guardia, calabozos, cuadra, horno y algibe del agua, sobre él se construyó una casamata para cañón y una plataforma para piezas móviles de montaña.

En toda esta construcción se cuidaron también ciertos detalles de belleza, entre los que sobresale la primera puerta de acceso, en forma de arco de herradura oval, con rosca almohadillada, en gusto directamente modernista, así como su paramento con pieras de corte pentagonal, va fechada en 1900; también se cuidaron las rejas de entrada al tunel de la caponera.

A lo largo de los distintos proyectos se descubre un proceso continuo de reducción de los edificios y de las obras en general; lo que motiva esta reducción son dos cuestiones simultáneas: por una parte el alto coste económico de las obras y por otra razones estratégicas, en orden a encontrar una perfecta protección de las construcciones por medio del escudo rocoso de la parte norte y a compensar el gasto y fortificación de Coll de Ladrones con el de La Sagüeta.

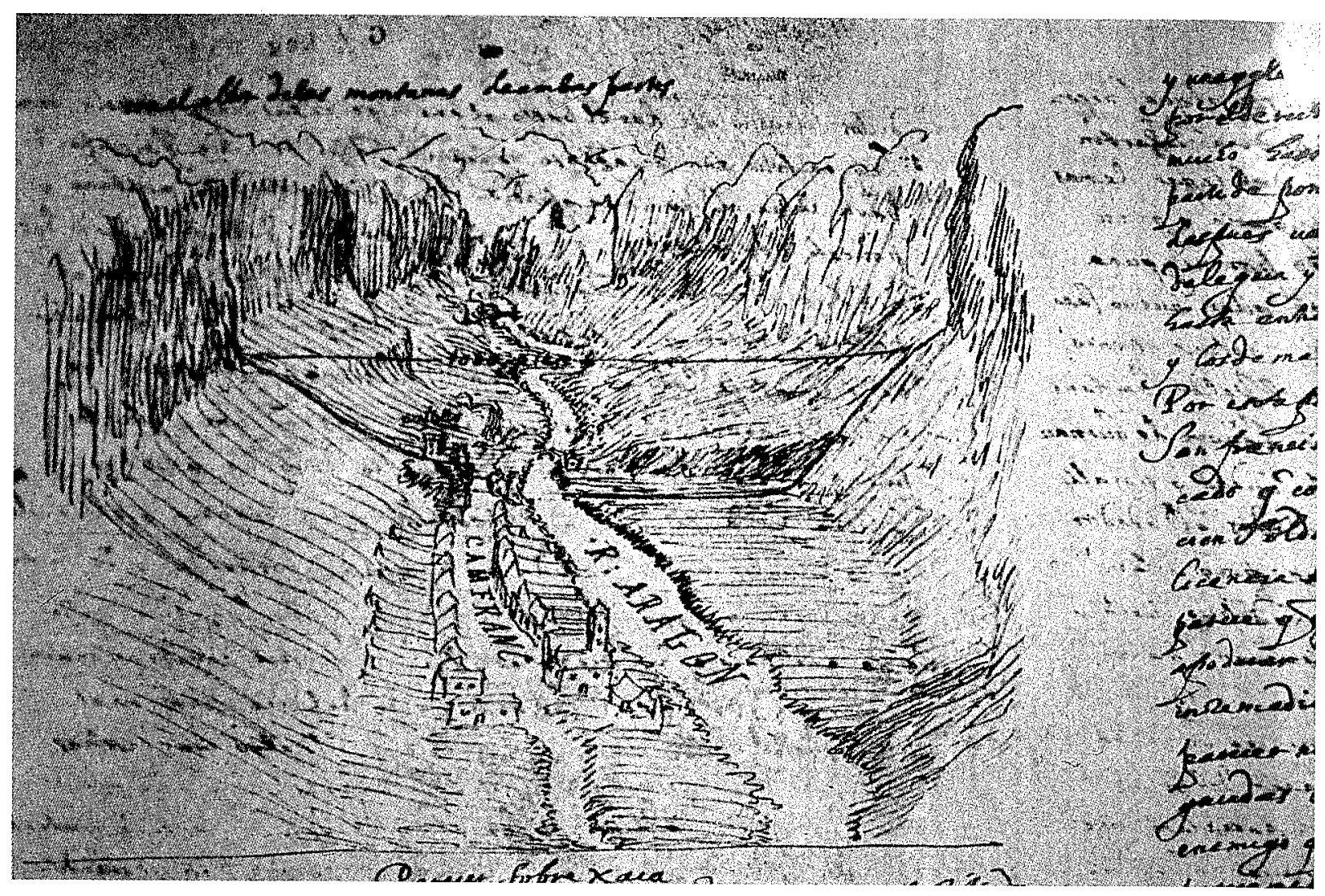

Lam. 1. Dibujo del valle de Canfranc por Spanochi. 


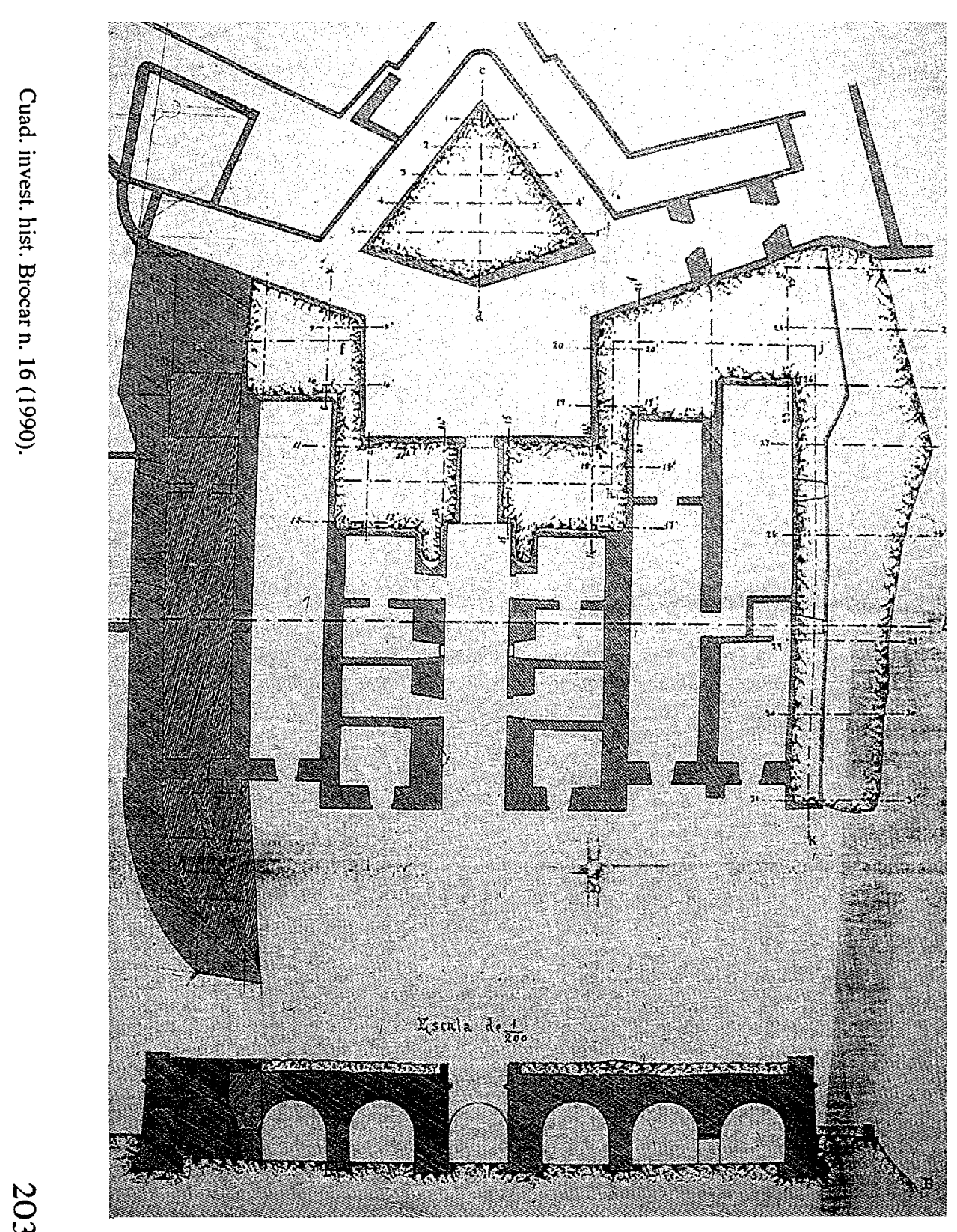

Lam. 2. Planta y corte del fuerte de Coll de Ladrones a principios del siglo XIX.

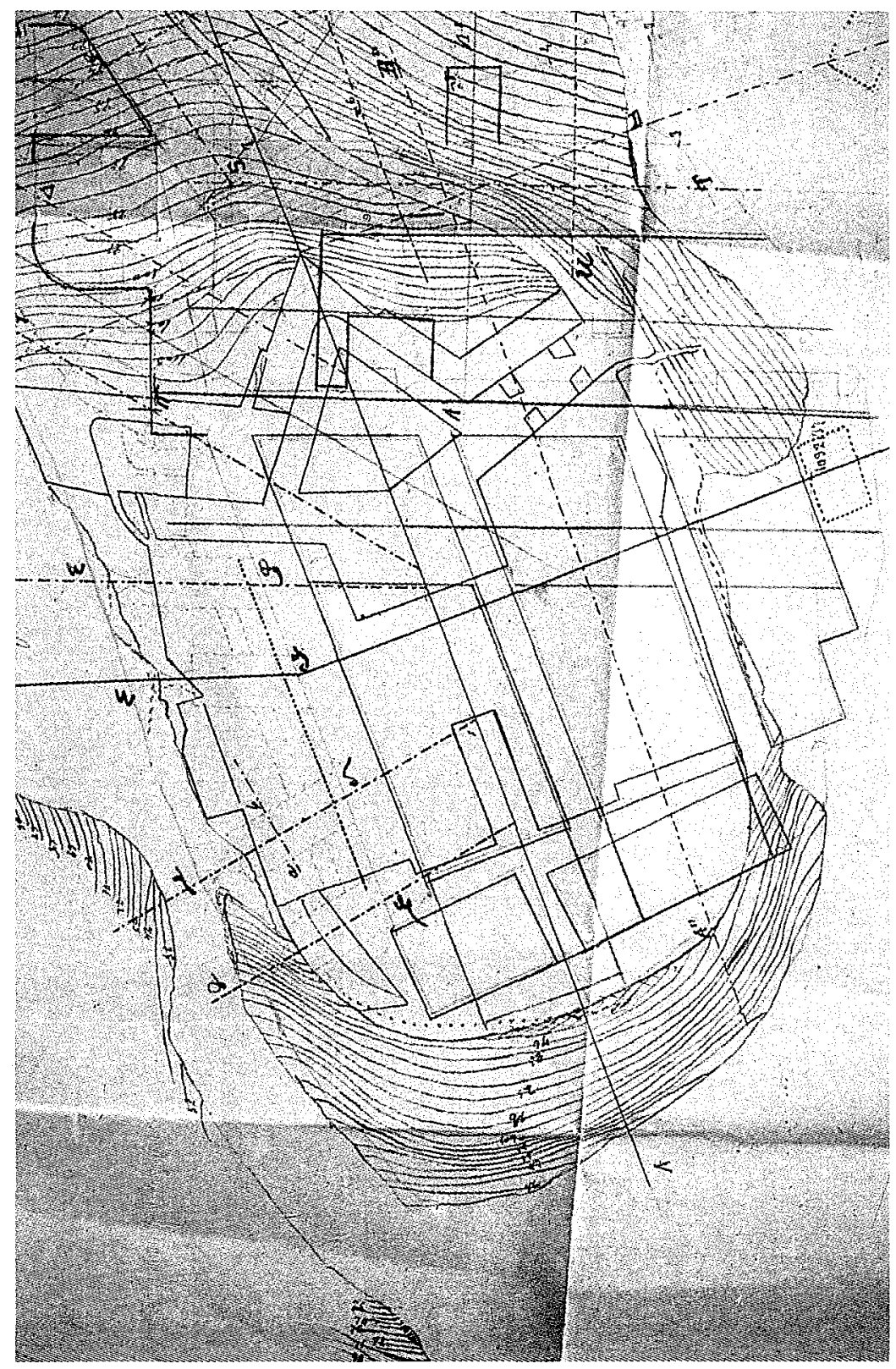

Lam. 3. Superposición de la planta de los diversos proyectos del siglo XIX donde se vé la reducción de las construcciones. 


\section{JUAN F. ESTEBAN}

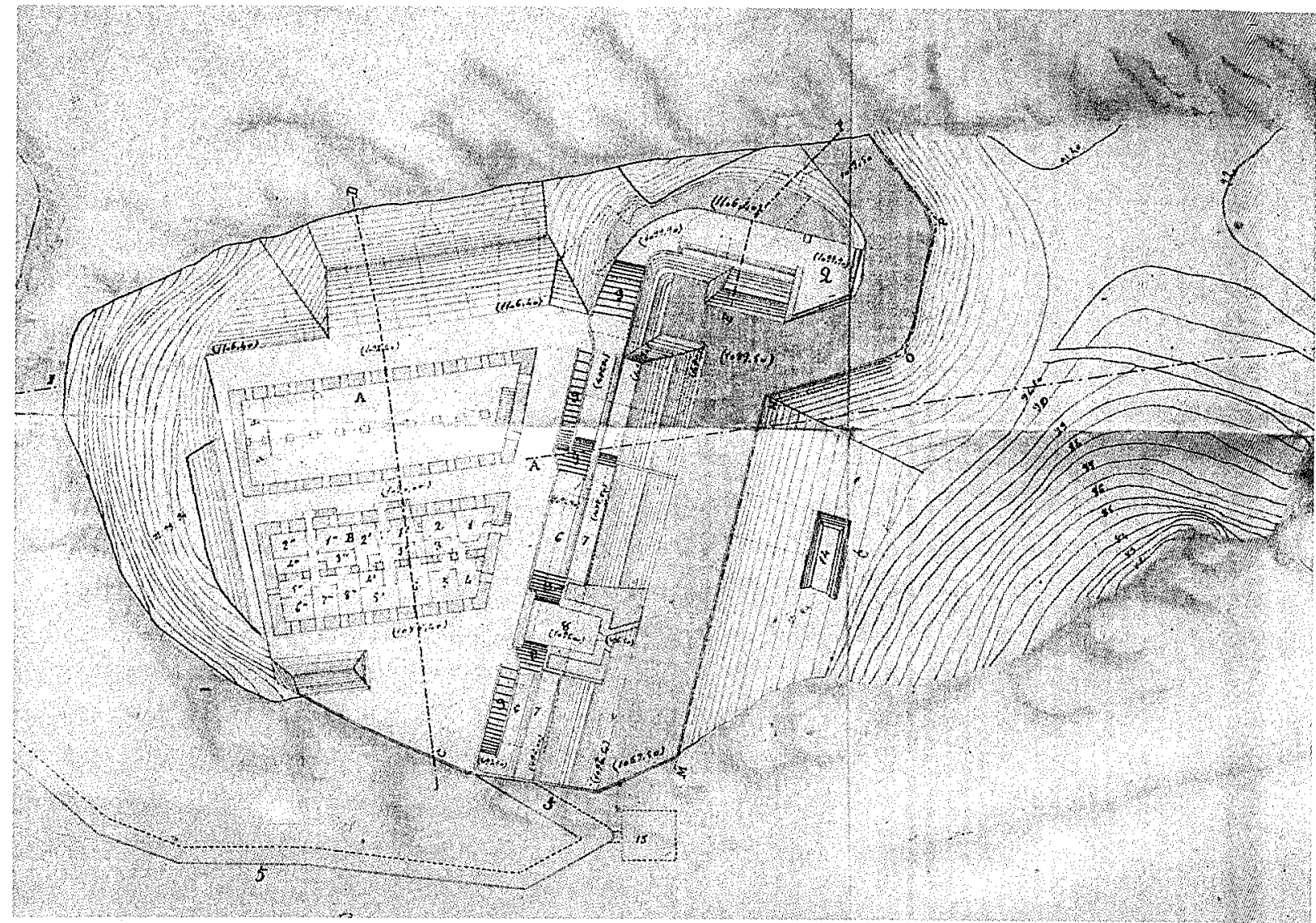

Lam. 4. Planta de Coll de Ladrones.

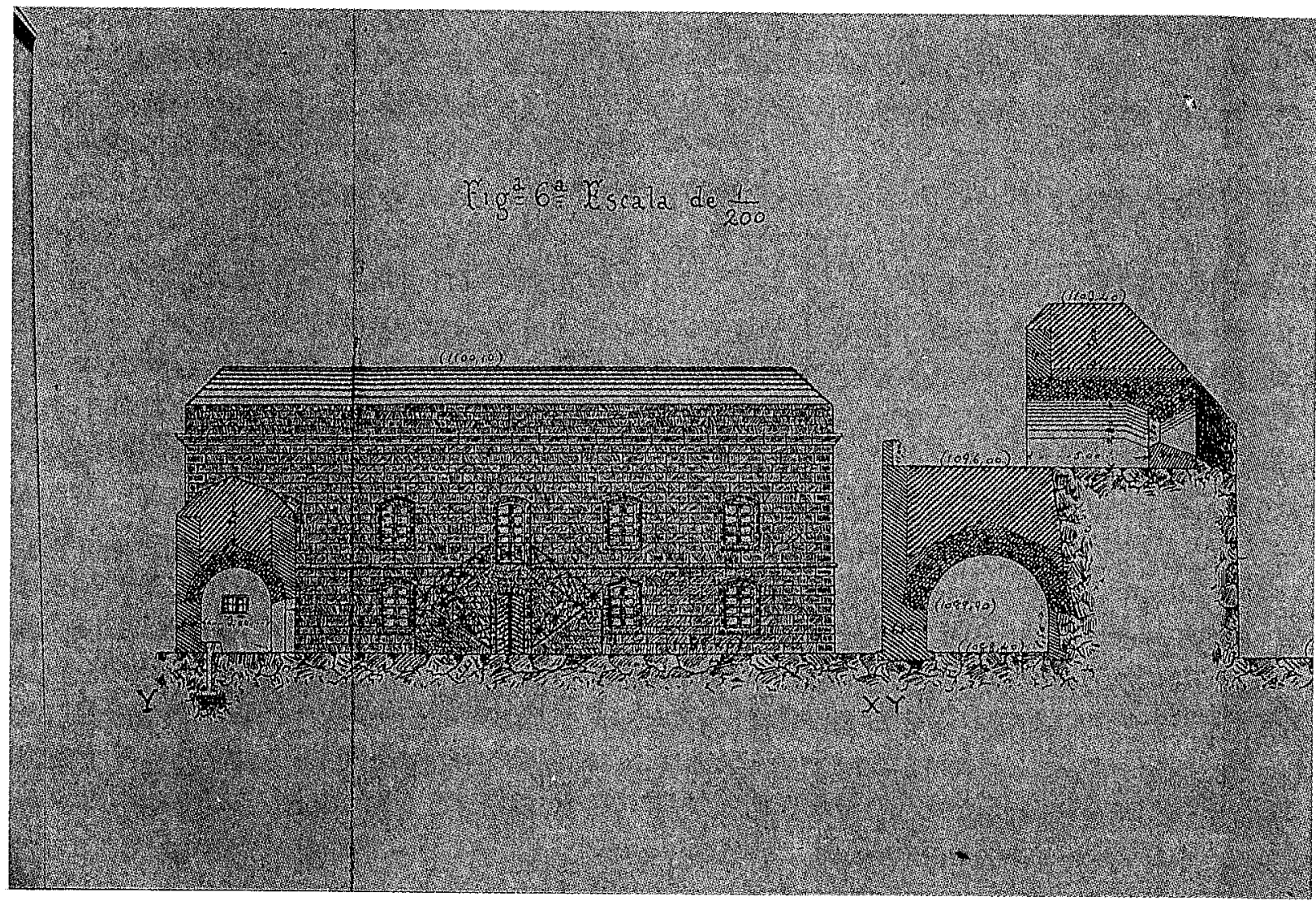

Lam. 5. Visión del fuerte de Coll de Ladrones, corte realizado por la fachada del edificio de "pabellones" 1888. 
PROCESO HISTORICO DE COLL DE LADRONES

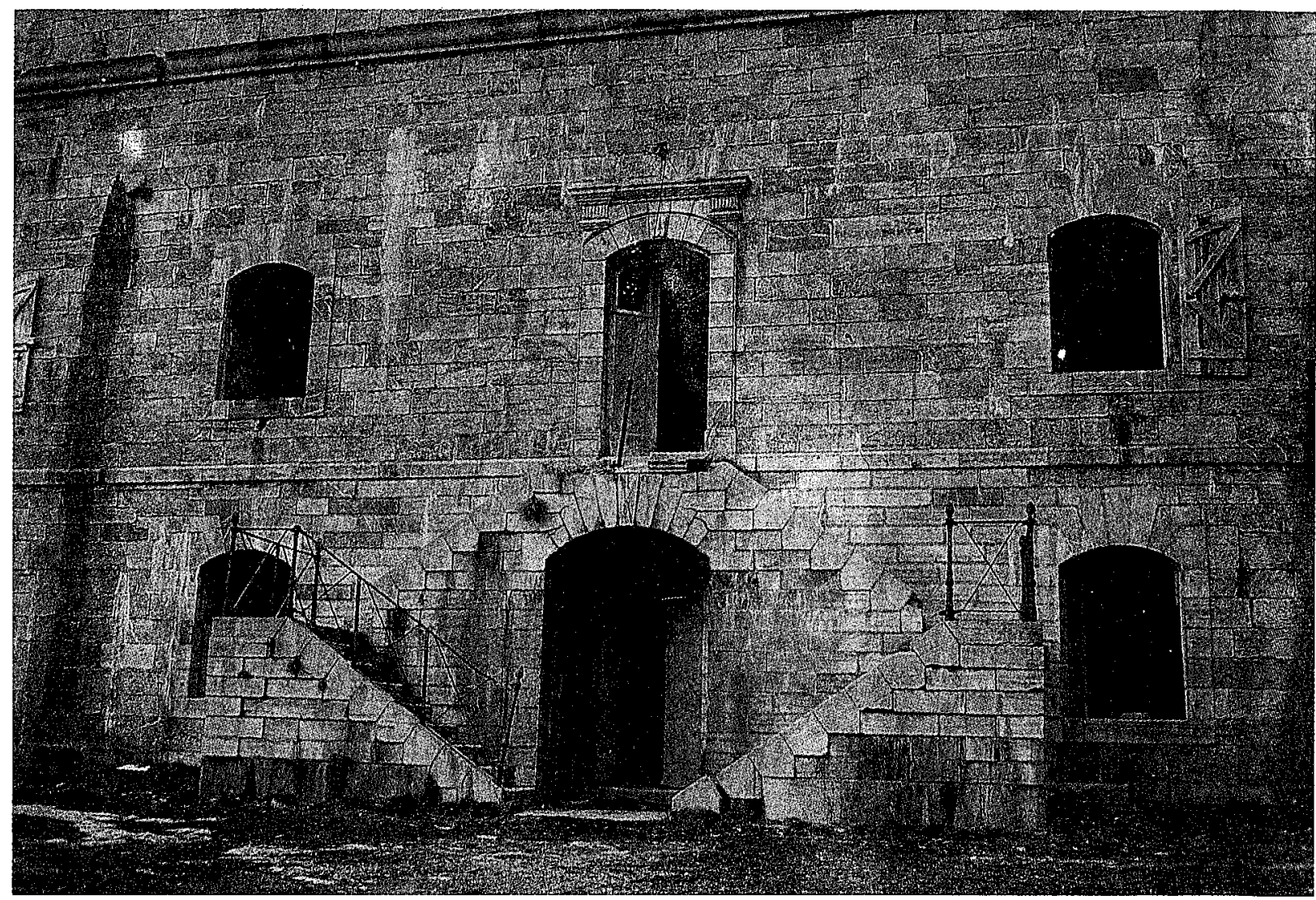

Lam. 6. Coll de Ladrones, pabellón principal.

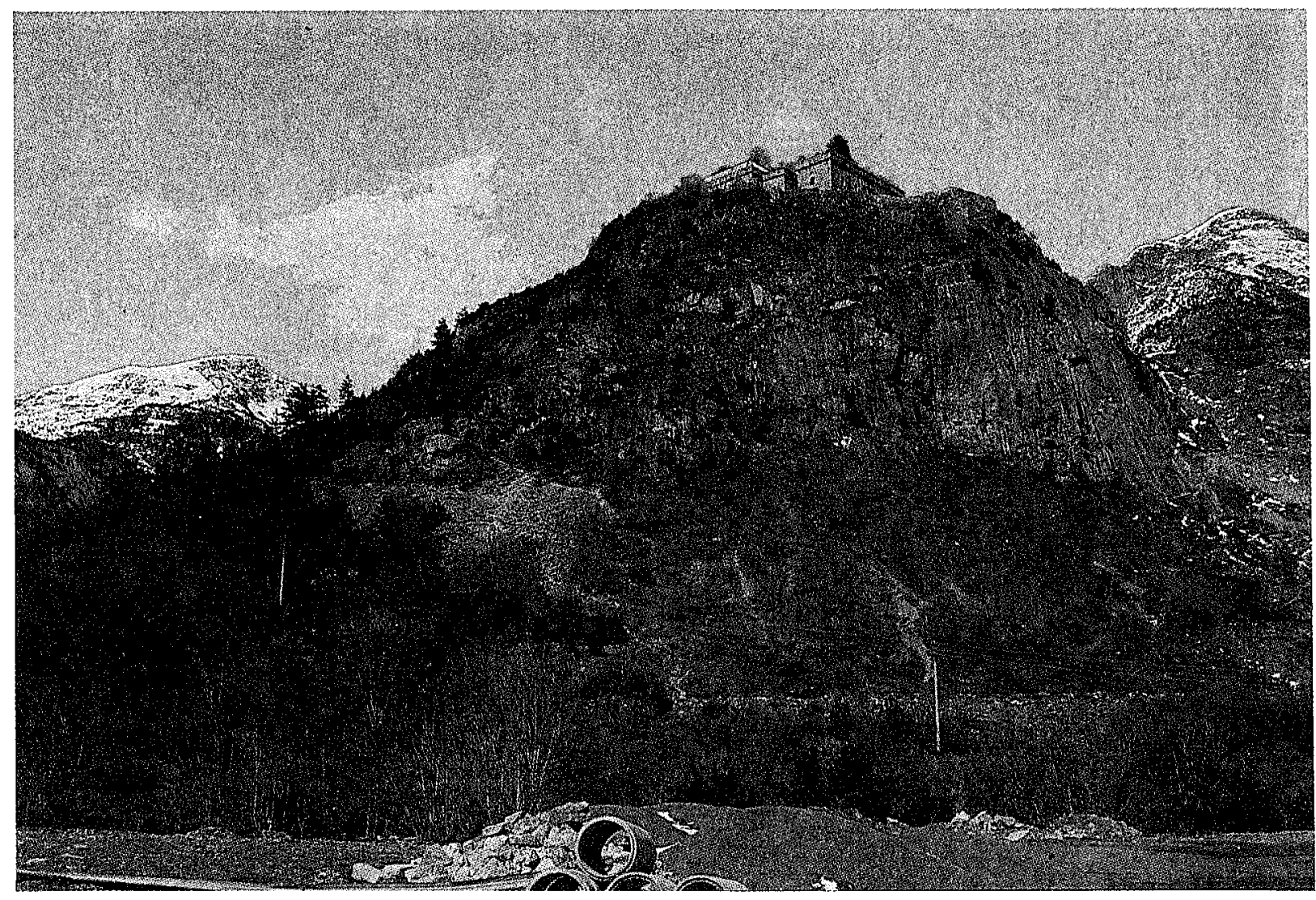

Lam. 7. Coll de Ladrones, emplazamiento. 
JUAN F. ESTEBAN

\section{Pi. 1 Enplaramierto para ametrallodora,}
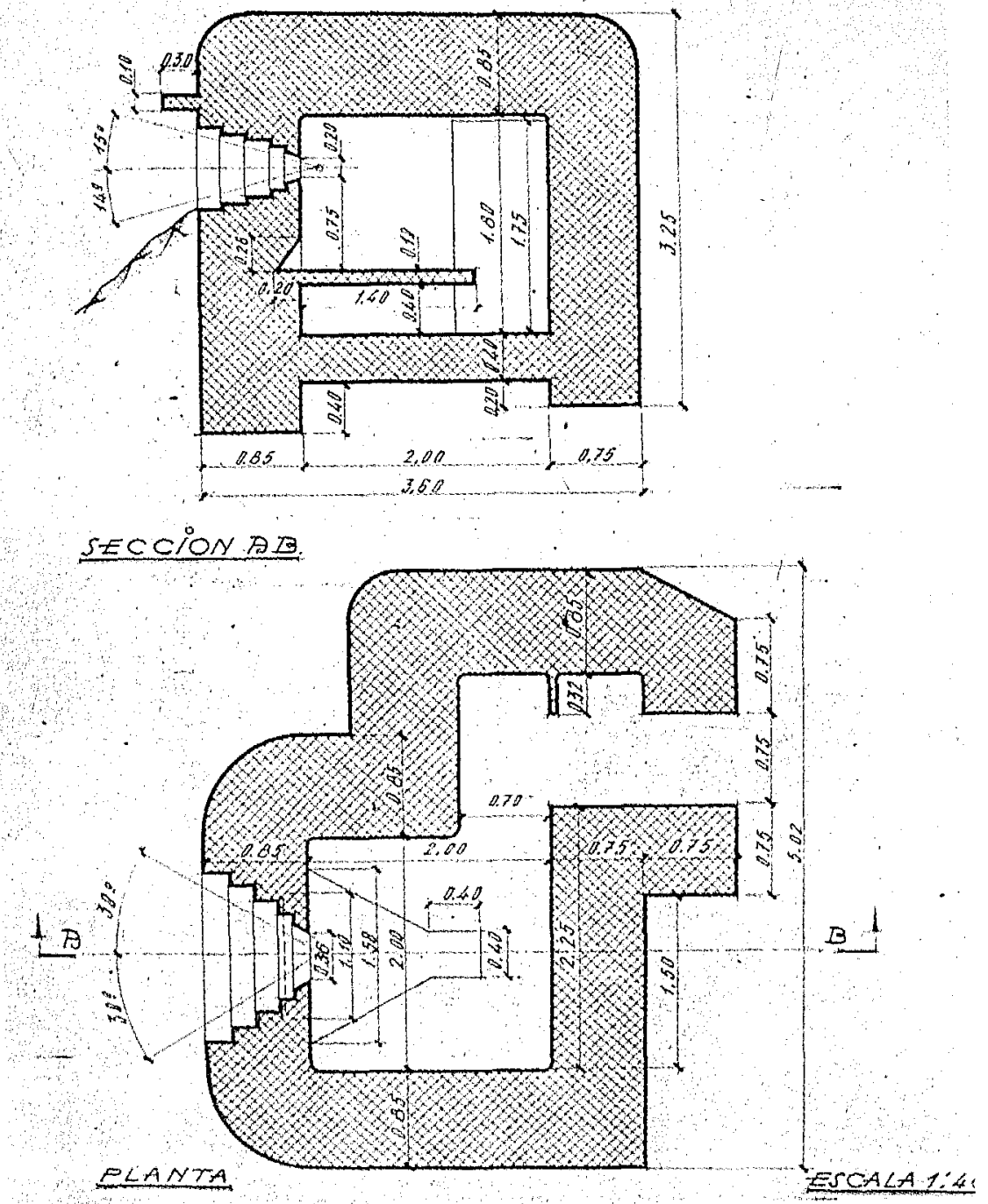

Lam. 8. Planta y alzado de un emplazamiento para ametralladora. 